\title{
Knowing when to assist: developmental issues in lifelong assistive robotics
}

\author{
Yiannis Demiris, Senior Member, IEEE
}

\begin{abstract}
Children and adults with sensorimotor disabilities can significantly increase their autonomy through the use of assistive robots. As the field progresses from short-term, taskspecific solutions to long-term, adaptive ones, new challenges are emerging. In this paper a lifelong methodological approach is presented, that attempts to balance the immediate contextspecific needs of the user, with the long-term effects that the robot's assistance can potentially have on the user's developmental trajectory.
\end{abstract}

\section{INTRODUCTION}

A SSISTIVE robots have been shown to provide significant increases in the autonomy of disabled users, with an immediate impact on their quality of life [1], but also have the potential to reduce the burden involved in caring for populations of disabled people in frequently under resourced clinical settings, such as rehabilitation and residential care centres. One of the most common methods for increasing autonomy is through the use of powered mobility, for example, powered wheelchairs. Rapid progress has been made in this field, enabling users with various types of disabilities to be able to control wheelchairs through various modalities, for example through EEG [2] or EMG [3] signals. Unfortunately, safety concerns frequently exclude potentially useful assistive powered wheelchairs for certain groups of patients, for example those with unilateral neglect [8], and many of disabled users find current power wheelchair control interfaces difficult to use [9].

An emerging challenge within this field is the design of methods that can incorporate developmental considerations in the algorithms that provide assistance to the user. For example, sensorimotor abilities develop rapidly during the first five years in children, and are necessary for enabling developing children to explore their physical environment and engage in meaningful social interaction. Children with disabilities, for example due to cerebral palsy [4], spinal muscular atrophy [5], or tetraplegia [6], are deprived from crucial opportunities to develop their cognitive and social skills, which has a negative impact on their overall continued development and quality of life. By providing powered mobility, and augmenting it with computational mechanisms for adaptive shared control we can facilitate socio-cognitive development, reduce "learned helplessness"

Manuscript received April 23, 2009.

Yiannis Demiris is with the Department of Electrical and Electronic Engineering, Imperial College London, South Kensington Campus, UK. e-mail: y.demiris@imperial.ac.uk, URL: http://www.iis.ee.ic.ac.uk/yiannis
[17], and assist in approximating the development pattern of the non-disabled population. Introducing powered mobility to young disabled children however is extremely challenging, and occupational therapists do not often consider it as an usable option. Common problems are postprovision difficulties with their use, for example learning initial control of the wheelchair, as well as a need to adjust operational parameters such as speed and sensitivity of control hardware according to the circumstances. In a national survey [7], 26 of 47 wheelchair service providers in England that had provided powered wheelchairs to children under the age of 5 reported post-provision difficulties, with 19 services specifically citing problems with the child learning initial control. Similar, although not identical, developmental considerations are important for adult cases, for example adult patients following stroke.

\section{TOWARDS CONDITIONALLY ASSISTIVE ROBOTS}

The fundamental point underlying our methodology is that assistive systems should not always and unconditionally assist the user, but attempt to balance the current needs of the user with the challenges that the user can learn to overcome with assistance from the robotic system. Requiring that assistive robots act as "intelligent tutors" shifts the focus to the developmental trajectory of the users, and allows a fruitful interaction between control engineering, as used in assistive robots, with other disciplines, including cognitive science and intelligent tutoring systems.

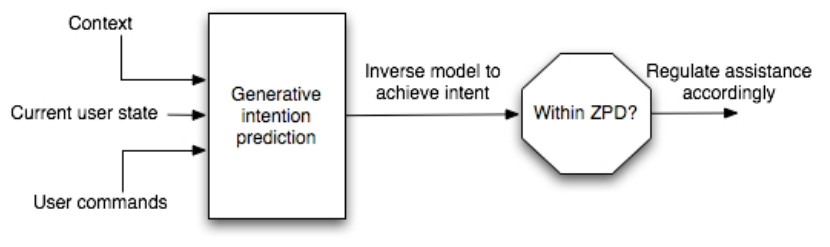

Figure 1: The overall architecture for regulating assistance

Figure 1 shows the overall assistive methodology we employ. The current context, state of the user, and any user commands are sent to the intention recognition module that attempts to infer what the user intends to do. A generative approach, using the HAMMER architecture [10] derives a set of inverse models (explained below) detailing how the intention can be achieved. The inverse models are examined 
as to whether they fall within the capabilities of the user (the Vygotskian concept of "Zone of Proximal Development" [18], or ZPD, discussed later), and the level of assistance is modulated accordingly. In the next few sections, the algorithms underlying each stage will be presented.

\section{A. Intention Prediction}

We use a generative approach to recognising the intentions of the user, using HAMMER (Hierarchical Attentive Multiple Models for Execution and Recognition) as the basis architecture [10], since it has proven useful in related Human Robot Interaction tasks, such as action interpretation [15] and imitation [16]. A brief review of the architecture:

Basic components: HAMMER uses the concepts of inverse and forward models. The inverse model receives as input an estimate of the current state of the controlled system and the desired target goal(s) and outputs the control commands that are needed to achieve that goal(s). A forward model of a system receives as inputs the current state of the system and a control command to be applied to it (as usually supplied by an inverse model) and outputs the predicted next state of the controlled system.

Controlling the robot: when HAMMER is asked to rehearse or execute a certain action to control the assistive robot (figure 2), the corresponding inverse model module is given information about the current state and, optionally, about the target goal(s). The inverse model then outputs the motor commands that are necessary to achieve these target goal(s). The forward model provides an estimate of the upcoming states should these motor commands be executed. The estimate can be compared with the target goal to produce a reinforcement signal for the inverse model depending on how much the model's motor commands brought the estimate closer to the target goal.

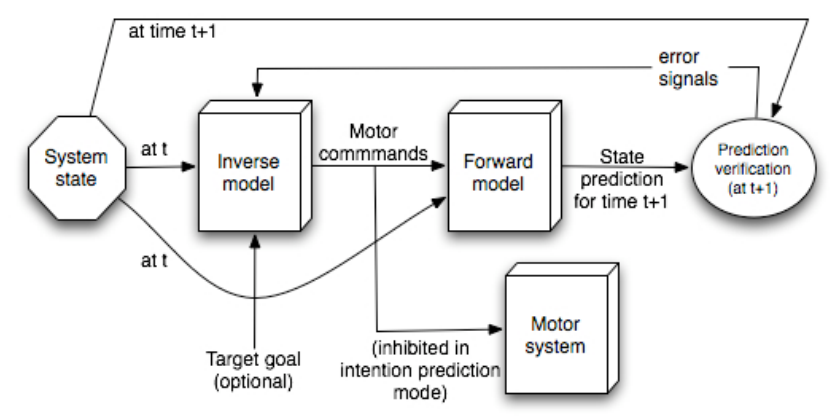

Figure 2: The building blocks of HAMMER; multiple paired inverse \& forward models of this form are maintained.

Predicting intentions: the HAMMER architecture uses an inverse-forward model coupling in a dual role: either for executing an action (as detailed before), or for recognising the same action if performed by a demonstrator/operator. When HAMMER operates in intention recognition mode, it can determine whether an ongoing action performed by a human user matches a particular inverse-forward model combination by feeding the human's current state as perceived by the observer system to the inverse model. The inverse model generates the motor commands that it would output if it was in that state and was executing the particular action. HAMMER consists of multiple pairs of inverse and forward models that operate in parallel. As the human user executes a particular action (with a particular unknown intention), and there are multiple models (possibilities) that can explain the ongoing states, we feed the perceived states into all of the system's available inverse models. This will result into the generation of multiple motor commands (representing the multiple hypotheses as to what action is being executed) that are sent to the forward models. The forward models generate predictions about the system's next state as described earlier and these are compared with the actual system's next state at the next time step. The error signals resulting from this comparison affect the confidence values of the inverse models. At the end of the action (or usually earlier as required in assistive control) the inverse model with the highest confidence value, i.e. the one that is the closest match to the operator's action is selected and is offered as an estimate of the user's intention. Importantly, the inverse models that are required in order to achieve the goal, are already calculated, and can be sent to the next module (ZPD) for evaluation.

\section{B. Zones of Proximal Development}

As explained earlier, our methodology is not to develop autonomous systems that will replace the user's control, nor constantly and unconditionally assist the user. Instead a lifelong approach is being developed that attempts to balance the current needs of the user with the challenges that the user could overcome with assistance from the robotic system. Principled methods to approach this task are in their infancy. Of fundamental importance is developing a method to approximate the user's current sensorimotor capabilities and potential for achieving their intentions with and without robotic help (akin to calculating the user's Zone of Proximal Development (ZPD), as put forward by Vygotsky [18]). While such concepts are widespread in intelligent tutoring systems where they are used potential strategies for helping (scaffolding) the user's development, they have not been operationalized before in the context of assistive robotics.

We use HAMMER's distributed networks of inverse and forward models [4] as a starting point for approximating the user's Zone of Proximal Development. The algorithm attempts to build hierarchical combinations of primitive inverse models that can solve a task, and compare them against the user's performance to determine the user's ZPD, and adjusts the level of assistance accordingly. The next few sections describe the theoretical aspects of this algorithm, 
and in particular how to quantify the current user performance and how to approximate the user's potential for achieving his/her intention.

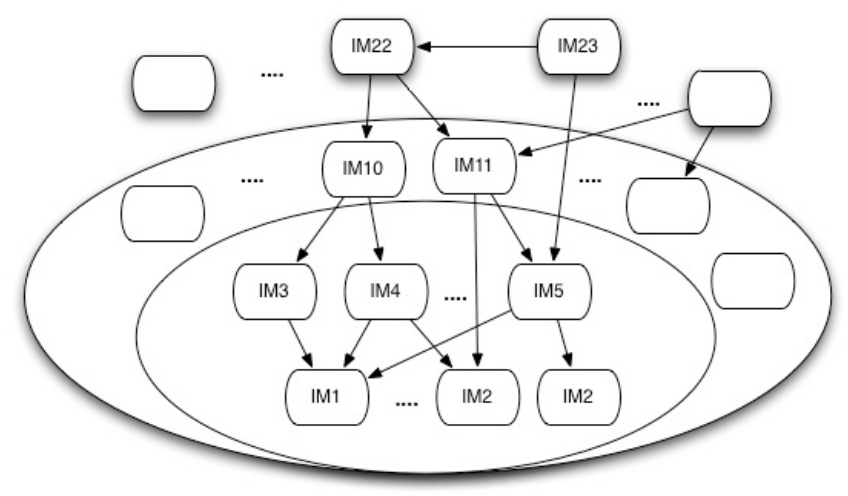

Figure 3: The zone of proximal development approximation using hierarchical inverse models (IM); in this conceptual example diagram, inverse models IM10 and IMI1 are within the ZPD since they rely on IMs 3, 4, 2, and 5 which have been successfully executed previously.

\section{The lower bound of the Zone of Proximal Development}

In our approach, in order to calculate the lower bound (inner circle) of the ZPD, i.e. in order to establish what the user is currently capable of, we use the amount of assistance that has been given by the shared controller supplementing the user's commands in the past as an approximation of these capabilities. The inverse models are annotated to indicate quantities and frequency of assistance given, as well as the effect it had on the overall user's performance.

\section{The upper bound of the Zone of Proximal Development}

Through the generative prediction of the user's intention, the HAMMER architecture has derived the inverse model (which we term Intended Task's Inverse Model (ITIM)) of what is required for the user to achieve his intention. To calculate whether the ITIM is within the user's ZPD, we proceed as follows:

- Decompose ITIM into the component inverse models that it requires for its completion.

- Retrieve the associated values for quantities and frequency of assistance given for the component inverse models in the past.

- Approximate the predicted level of combined shared control that needs to be given. For this, either use heuristics (for example, if a number of component inverse models are organised serially, take the highest quantity of assistance among the components as approximation, while if there are components organised in parallel (need to be executed concurrently), use a weighted sum of the components as approximation), or learn this by observation.
- Determine whether this falls within acceptable levels to qualify it as within the ZPD of the user. The threshold can be user- or practitioner-defined.

\section{Regulating assistance}

Once we have determined whether the intended task (ITIM) falls within the user's ZPD, the challenge that remains is to adapt the human-robot interface options and levels of shared control to facilitate execution. The challenge here is to maximise not only short-term benefits (success in the current task) but also long term development (such as deterioration of morale due to lack of opportunities to overcome challenges). Options include:

- A user-defined level of assistance that could be given by default throughout all tasks (which the user can easily adjust to suit his current needs and desires)

- A situation-dependent level of assistance, that can factor in the user's emotional state, contextual aspects (such as whether lack of assistance will pose a danger to self or others, or whether it will affect the perceptions of others towards the user (for e.g. assistance might be increased in public places and reduced in private settings).

- Disability specific training needs, potentially taking into consideration observations as to whether it was effective.

- Approaches that balance the last two options, based on practitioners' defined developmental or rehabilitation schedules.

\section{AN ASSISTIVE WHEELCHAIR EXAMPLE}

Up to now we have deliberately remained at the architectural level, as to avoid making the algorithms specific to only one type of assistive robot. In this section we will briefly describe the operationalization of these algorithms on an assistive robotic wheelchair, to demonstrate this process.

Figure 4 shows our experimental setup: a powered wheelchair has been adapted for computer control; human commands are sent through the joystick to the shared control system operating on a tablet PC, which estimates (through the wheelchair's cameras and laser scanners) whether the resulting action will have the intended effect, and be safe for the user, and alters the commands appropriately $[11,12]$; a wearable eye-gaze tracker evaluates the effects the assistance has on the attention patterns of the user [14]. In variations of our experiments, the user is controlling the wheelchair through EOG/EMG signals.

The user's current performance is evaluated through a number of criteria, for example, the smoothness of the user's joystick movements $[11,12]$, or the amount of assistance that was required for a particular inverse model to be successful, among others. For example, the user is presumed to have mastered a particular competence such as traversing a door when the number and frequency of corrective movements 
in Proceedings of the 31st Annual International Conference of the IEEE Engineering in Medicine and Biology Society (EMBC 2009), pp 3357-3360, Minneapolis, Minnesota, USA, September 2-6, 2009.

has dropped below a certain level. Throughout our experiments $[11,12,14]$, having the adaptive shared control system significantly improved the performance of the wheelchair users, for example by improving the smoothness of their joystick movements.

Of crucial importance to the adoption of these algorithms is their evaluation. In one of our studies [14], we used a headmounted eye-gaze tracker to record patterns of eye activity during operation of the assistive wheelchair with and without shared control, which revealed differences in the eye-gaze patterns of users when they were assisted, with the latter patterns being more varied [14]. This emphasizes an important aspect of lifelong assistive robots: in addition to the development of the user model by the assistive robot, the user is concurrently forming a mental model [13] of the behavior of robot and the assistance s/he can expect from it; the two models co-develop and their dynamics are interlinked, putting an additional constraint on the amount of disturbance we can impose on either. An important challenge to our approach is designing suitable interfaces that can explain to the user the robot's view of his/her capabilities, as well as the intended level of assistance.

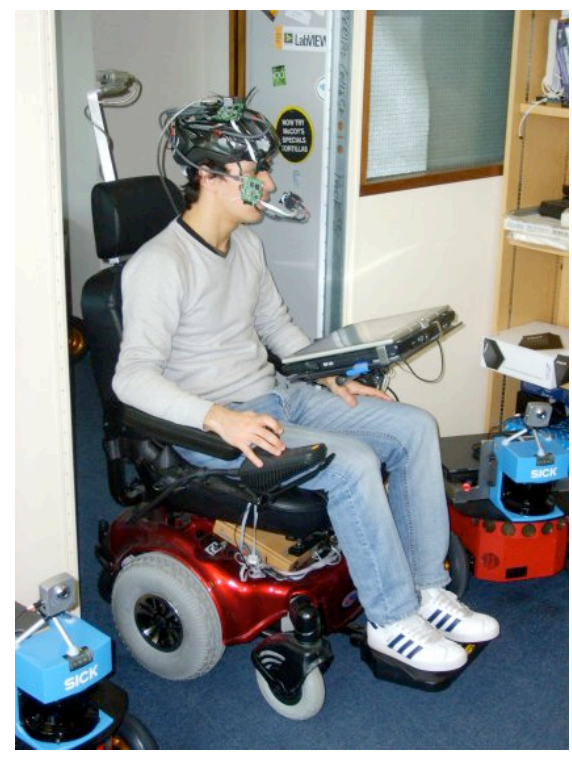

Figure 4: The assistive wheelchair experimental setup

\section{EPILOGUE}

As the technological sophistication of assistive robots increases, additional demands will be placed upon them. They will need to move beyond unconditional assisting towards conditionally assisting (or potentially even hindering) the user as to improve their developmental trajectory. We have outlined our methodology that attempts to bring concepts from cognitive science, developmental psychology and intelligent tutoring systems into the assistive robotics field. In addition to the usefulness that such concepts bring to the field of assistive robotics, their operationalisation in robotic systems can help clarify and sharpen their theoretical details.

\section{ACKNOWLEDGMENT}

Thanks to Tom Carlson, Murilo Fernandes Martins, Mireno Rossi and Valerio Chang, for their help with the robotic experiments.

\section{REFERENCES}

[1] A. Davies, L. H. De Souza, and A. O. Frank, "Changes in the quality of life in severely disabled people following provision of powered indoor/outdoor chairs", Disability and Rehabiliation, 25(6):286-290 (2003).

[2] F. Galán, M. Nuttin, E. Lew, PW Ferrez, G Vanacker, J Philips , J del R Millán, "A brain-actuated wheelchair: asynchronous and noninvasive Brain-computer interfaces for continuous control of robots", Clinical Neurophysiology, 119(9): 2159-69 (2008).

[3] Jeong-Su Han, Zenn Bien, Z.; Dae-Jin Kim; Hyong-Euk Lee; JongSung Kim, "Human-machine interface for wheelchair control with EMG and its evaluation," Proceedings of the 25th Annual International Conference of the IEEE on Engineering in Medicine and Biology Society, 2003. vol.2, pp. 1602-1605 Vol.2, 17-21 Sept. 2003

[4] J. Furumasu, P. Guerette and D. Tefft, "Relevance of the Paediatric Powered Wheelchair Screening Test for children with cerebral palsy", Developmental Medicine and Child Neurology, 46: 468-474 (2004).

[5] M. A. Jones, I. R. McEwen and L. Hansen, "Use of Powered Mobility for a Young Child with Spinal Muscular Atrophy", Physical Therapy, 83(3): 253-262 (2003).

[6] M. Bottos, C. Bolcati, L. Sciuto, C. Ruggeri, and A. Feliciangeli, "Powered wheelchairs and independence in young people with tetraplegia", Developmental Medicine and Child Neurology, 43:769777 (2001).

[7] J. Nicholson and M. Bonsall, "Powered Mobility for Children under Five Years of Age in England", British Journal of Occupational Therapy, 65(5):291-293 (2002)

[8] J. Dawson and H. Thornton, "Can Patients with Unilateral Neglect following Stroke drive Electrically Powered Wheelchairs?", British Journal of Occupational Therapy, 66(11):496-504 (2003).

[9] L. Fehr, W. E. Langbein, and S. B. Skaar, "Adequacy of power wheelchair control interfaces for persons with several disabilities: A clinical survey", Journal of Rehabilitation Research and Development, 37(3): 353-360 (2000).

[10] Y. Demiris, "Prediction of intent in robotics and multi-agent systems", in Cognitive Processing 8: 151-158, 2007.

[11] T. Carlson and Y. Demiris, "Human-Wheelchair Collaboration through Prediction of Intention and Adaptive Assistance", Proceedings of IEEE International Conference on Robotics \& Automation, CA, USA, pp 3926-3931 (2008)

[12] T. Carlson and Y. Demiris, "Collaborative Control in Human Wheelchair Interaction Reduces the Need for Dexterity in Precise Manoeuvres", In Proc. of ACM/IEEE HRI-2008 workshop on "Robotic Helpers: User Interaction, Interfaces and Companions in Assistive and Therapy Robotics", Amsterdam, Netherlands, pp 59-66, (2008).

[13] D. Gentner, "Psychology of mental models", in N. J. Smelser and P. B. Bates, editors, International Encyclopedia of the Social and Behavioral Sciences, pages 9683-9687, Elsevier, Amsterdam, (2002).

[14] T. Carlson and Y. Demiris, "Using Visual Attention to Evaluate Collaborative Control Architectures for Human Robot Interaction", Proceedings of the AISB HRI Symposium, Edinburgh, Scotland, to appear (2009).

[15] Y. Demiris and B. Khadhouri, "Hierarchical Attentive Multiple Models for Execution and Recognition (HAMMER)", in Robotics and Autonomous Systems, 54:361-369, 2006.

[16] Y. Demiris and A. Meltzoff, "The Robot in the Crib: A developmental analysis of imitation skills in infants and robots", Infant and Child Development, 17:43-53, 2008.

[17] C. Butler, "Augmentative mobility. Why do it?", Physical Medicine and Rehabilitation Clinics of North America, 2 (4), 801-815, 1991.

[18] L.S. Vygotsky, "Mind in society", Cambridge, MA: Harvard University Press, 1978. 